\title{
Transcriptome analysis of smut fungi reveals widespread intergenic transcription and conserved antisense transcript expression
}

\author{
Michael E. Donaldson ${ }^{1 \dagger}$, Lauren A. Ostrowski ${ }^{1,3+}$, Kristi M. Goulet ${ }^{1}$ and Barry J. Saville ${ }^{1,2^{*}}$
}

\begin{abstract}
Background: Biotrophic fungal plant pathogens cause billions of dollars in losses to North American crops annually. The model for functional investigation of these fungi is Ustilago maydis. Its $20.5 \mathrm{Mb}$ annotated genome sequence has been an excellent resource for investigating biotrophic plant pathogenesis. Expressed-sequence tag libraries and microarray hybridizations have provided insight regarding the type of transcripts produced by $U$. maydis but these analyses were not comprehensive and there were insufficient data for transcriptome comparison to other smut fungi. To improve transcriptome annotation and enable comparative analyses, comprehensive strand-specific RNA-seq was performed on cell-types of three related smut species: U. maydis (common smut of corn), Ustilago hordei (covered smut of barley), and Sporisorium reilianum (head smut of corn).
\end{abstract}

Results: In total, > 1 billion paired-end sequence reads were obtained from haploid cell, dikaryon and teliospore RNA of U. maydis, haploid cell RNA of U. hordei, and haploid and dikaryon cell RNA of S. reilianum. The sequences were assembled into transfrags using Trinity, and updated gene models were created using PASA and categorized with Cufflinks Cuffcompare. Representative genes that were predicted for the first time with these RNA-seq analyses and genes with novel annotation features were independently assessed by reverse transcriptase PCR. The analyses indicate hundreds more predicted proteins, relative to the previous genome annotation, could be produced by U. maydis from altered transcript forms, and that the number of non-coding RNAs produced, including transcribed intergenic sequences and natural antisense transcripts, approximately equals the number of mRNAs. This high representation of non-coding RNAs appears to be a conserved feature of the smut fungi regardless of whether they have RNA interference machinery. Approximately 50\% of the identified NATs were conserved among the smut fungi.

Conclusions: Overall, these analyses revealed: 1) smut genomes encode a number of transcriptional units that is twice the number of annotated protein-coding genes, 2) a small number of intergenic transcripts may encode proteins with characteristics of fungal effectors, 3) the vast majority of intergenic and antisense transcripts do not contain ORFs, 4) a large proportion of the identified antisense transcripts were detected at orthologous loci among the smut fungi, and 5) there is an enrichment of functional categories among orthologous loci that suggests antisense RNAs could have a genome-wide, non-RNAi-mediated, influence on gene expression in smut fungi.

Keywords: Smut fungi, RNA-seq, Non-coding RNAs, Natural antisense transcripts, Ustilago maydis, Ustilago hordei, Sporisorium reilianum

\footnotetext{
* Correspondence: barrysaville@trentu.ca

'Equal contributors

'Environmental and Life Sciences Graduate Program, Trent University,

Peterborough K9L 0G2, ON, Canada

${ }^{2}$ Forensic Science Program, Trent University, Peterborough K9L 0G2, ON,

Canada

Full list of author information is available at the end of the article
} 


\section{Background}

Basidiomycete biotrophic pathogens are a major threat to cereal crop production world-wide. Many of these fungi are obligate biotrophs and not readily amenable to molecular analysis. In contrast, the corn smut fungus Ustilago maydis can be cultured in defined conditions, is amenable to biochemical and genetic analysis as well as molecular manipulation, and its genome has been sequenced and annotated [1]. To improve the resources available for using this model, we have created and analyzed deep RNA-seq libraries that will enhance genome annotation. In other classes of phytopathogenic filamentous fungi, similar analyses have enabled the detection of previously unidentified transcripts, and increased the accuracy and coverage of transcriptome annotations. Several studies in the past 2 years alone have employed high-throughput and massively parallel RNA-seq approaches to update current genome annotations of phytopathogenic filamentous fungi including those of Botrytis cinerea (504 new gene models; [2]); Colletotrichum graminicola (906 new and 819 updated gene models; [3]) and Fusarium graminearum (412 new and 1529 updated gene models; [4]). These studies and others have been instrumental in correcting annotation errors, gathering information on untranslated regions (UTRs) and alternative splice sites, as well as identifying novel protein-coding genes and new isoforms, contributing to our understanding of pathogenicity determinants in phytopathogenic fungi. The deep transcriptome analysis presented here involved $U$. maydis, $U$. hordei, and Sporisorium reilianum and was carried out with a focus on comparative analyses which expanded the genome annotations and identified conserved natural antisense transcripts.

$U$. maydis was the first biotrophic plant pathogen to have its genome sequenced [1]. This initial sequencing study generated a $20.5 \mathrm{Mb}$ genome sequence containing 6902 protein-coding gene annotations; but, introns were annotated for only $30 \%$ of protein-coding genes, and UTR data were not provided. Very few previously-characterized pathogenesis genes were identified, however 426 secreted proteins were predicted. A function could not be ascribed to $298(70 \%)$ of these, and $193(65 \%)$ were found exclusively in $U$. maydis. These secreted proteins included potential effector proteins which stimulated their functional analysis and provided tremendous insight into $U$. maydis pathogenesis (e.g. [5-7]). Further information on secreted proteins was obtained by comparative genome analyses [8,9], however these comparisons also utilized the initial $U$. maydis genome annotation and did not include information from transcriptome analyses. Earlier expressed-sequence tag (EST) library investigations [10-13] identified novel protein-coding genes, cell-type-specific gene expression profiles, and alternative splicing. They also improved existing gene structure annotations and determined 5' and 3' UTR lengths [10, 14]. Together, these transcriptome-focused analyses substantially improved the initial annotation of protein-coding genes in $U$. maydis, providing an improved dataset for functional analysis.

In addition to improving our understanding of the protein-coding genome, EST analyses revealed noncoding transcription in $U$. maydis. Of the 6284 predicted gene locations identified by 24643 ESTs, 800 (13\%) aligned to regions of the genome not recognized as coding in the original annotation [10]. Hundreds of noncoding RNAs (ncRNAs), including natural antisense transcripts (NATs), were identified [10, 11, 15]. NATs are long ncRNAs transcribed from the strand opposite to a protein-coding transcript, thus exhibiting sequence complementarity to mRNAs. Several fungal NATs and other ncRNAs are developmentally regulated or expressed in a cell-type-specific manner [15, 16], and functional roles for such transcripts have been elucidated (Reviewed in $[17,18]$ ). Roles in the regulation of pathogenesis and metabolism were revealed by the characterization of a ncRNA and several NATs [11, 15, 19]. The conservation of these transcripts in the related smut species $U$. hordei and/or $S$. reilianum, suggested that conservation of ncRNA/NAT function was also possible $[11,15,19]$.

In the past 3 years, transcriptome-wide ncRNA detection in phytopathogenic filamentous fungi has surged with the following findings: 121 ncRNAs in F. graminearum [4], 187 small RNAs in Puccinia striiformis f.sp. tritici [20], 120 ncRNAs in C. graminicola [3], 155 ncRNAs in Rhizoctonia solani [21], and 5394 ncRNAs in Zymoseptoria tritici [22]. Novel NATs have been identified in species including B. cinerea (30 spliced NATs; [2]) and $U$. maydis (292 NATs; [15]). In $U$. maydis, altering the level of ncRNAs and NATs through promoter deletion reduced virulence $[11,15]$. Together these studies indicate that ncRNAs and NATs contribute to the control of pathogenic development in fungi but provide no information on whether these functions are conserved or unique in different fungal species.

$U$. maydis, $U$. hordei, and S. reilianum share common characteristics such as the production of pathogenic dikaryotic filaments as a result of compatible saprophytic haploid cell fusion, proliferation within agronomically important crops, and the production of teliospores as protective dispersal agents [23]. The three species differ in that $U$. maydis infects maize, with localized symptom development [1], whereas $U$. hordei and S. reilianum systemically infect barley/oats and maize/sorghum, respectively, eliciting symptoms only in their reproductive structures $[8,9]$. Investigations into the molecular basis of these host preference and growth form differences have focused on the identification of distinct effector proteins $[12,16,17]$. However, it is unlikely that 
this is the only form of variation leading to these differences; here we focus on deep transcriptome analysis of the best characterized smut fungus, $U$. maydis, while augmenting the information obtained through comparative transcriptome analyses. One major difference between these fungi is that $U$. maydis lacks RNAi machinery, while $U$. hordei and $S$. reilianum retain this machinery $[9,24]$. Given that Dicer can process long ncRNAs including NATs in filamentous fungi (e.g. [25]), and that long intergenic ncRNAs (lincRNAs) and NATs are known to regulate $U$. maydis gene expression and pathogenesis $[11,15,19]$, it is possible that some of the roles of these transcripts in the respective fungi are distinct. If this was the case, these distinct functions could differentially influence gene expression among the smut fungi and contribute to host specificity differences. It is also possible that subtle differences in conserved antisense functions could contribute to variation in pathogenic lifestyle. To begin to investigate these possibilities, the lincRNAs, including NATs in these fungi, must be comprehensively identified.

The existing transcriptome data on $U$. maydis, $U$. hor$d e i$, and S. reilianum were not collected in a manner that focused on which DNA strands were represented in the transcriptome, nor was there an effort to identify ncRNA expression. The smut fungus transcriptome analyses presented herein utilized massively parallel stranded RNA-seq to update current annotations and provide comprehensive identification and characterization of novel $U$. maydis protein-coding isoforms, lincRNAs, NATs, and their putative orthologs in the other smuts. The identification of a large number of previously unrecognized transcripts provides a critical knowledge base for a more complete understanding of effectors, and the discovery of a large number of lincRNAs and NATs that could influence gene expression and pathogenesis in $U$. maydis as well as the related smut fungi.

\section{Results}

\section{RNA-seq library creation and RNA-seq based transcript} characterization

Compatible haploid cells from each smut species (Table 1) were grown separately, or incubated together to form dikaryons or, for $U$. maydis, co-injected into corn where they fused to form the dikaryon and eventually produced diploid teliospores. RNA was isolated from haploids, dikaryons and teliospores for RNA-seq library creation (Additional file 1). Strand-specific RNA-seq libraries were generated and deep Illumina RNAsequencing yielded $>1$ billion paired-end reads ( $\sim 95$ million paired-end reads per library; Additional file 2).

Paired-end reads were pooled for each fungus and transfrags were predicted using Trinity [26]. Existing Munich Information Centre for Protein Sequences
Table 1 Fungal strains used in this study

\begin{tabular}{lll}
\hline Strain & Genotype & Source \\
\hline Ustilago maydis & $a 2$ b2 & {$[59]$} \\
518 & $a 1$ b1 & {$[59]$} \\
$521^{\text {a }}$ & & \\
Ustilago hordei & MAT-1 & {$[60]$} \\
Uh 4857-4 (alias Uh364) & MAT-2 & {$[60]$} \\
Uh 4857-5 (alias Uh365) & & \\
Sporisorium reilianum & $a 1$ b1 & {$[61]$} \\
SRZ1 & a2 b2 & {$[61]$} \\
SRZ2 $^{\text {a }}$ &
\end{tabular}

${ }^{\mathrm{a}}$ Reference strain used for genome sequencing

(MIPS) gene models were updated to include 5' and 3' UTRs, alternative splice isoforms, and gene model fusions using the program to assemble spliced alignments (PASA; [27]). Predicted gene models were categorized by Cufflinks Cuffcompare ('e'= possible pre-mRNA fragment; ' $\mathrm{i}$ '= transfrag entirely within reference intron; ' $j$ ' = potentially novel isoform with at least one splice junction shared with reference transcript; ' $o$ ' = generic exonic overlap with a reference transcript; ' $\mathrm{p}$ ' = potential polymerase run-on fragment; ' $x$ ' = antisense transcript; ' $u$ ' = intergenic transcript). The numbers of identified annotation features for each fungus are presented in Table 2. Detailed genome annotation for each fungus is provided in GFF3-formatted .txt files (Additional files 3, 4 and 5). UTR annotations were determined for $87 \%$ of $U$. maydis, $79 \%$ of $U$. hordei, and $88 \%$ of $S$. reilianum genes; and a low number of UTRs in each of the three fungi contained introns (Table 2). Average UTR lengths $\left(5^{\prime} / 3^{\prime}\right)$ were calculated as $289 \mathrm{bp} / 304 \mathrm{bp}$ for $U$. maydis, $235 \mathrm{bp} / 231 \mathrm{bp}$ for $U$. hordei, and $211 \mathrm{bp} /$ 180 bp for S. reilianum genes. 763, 681, and 466 alternate splice isoforms were predicted using PASA for $U$. maydis, $U$. hordei, and S. reilianum genes, respectively (Table 2). The PASA-predicted isoforms differ from those annotated by Cufflinks Cuffcompare ([28]; classes 'e,' 'j', and 'o') because by default, PASA was set to ignore alternate splice isoforms whose coding sequence was altered by $>30 \%$ in length. In an effort to be consistent with previous MIPS gene formatting, these PASA-predicted isoforms were designated as "gene name - $\mathrm{N}$ ", where $\mathrm{N}=$ an alphabetized letter starting with "-B" (e.g. UMAG_02775-B). Further, only 10,13 , and 14 merged transcripts were predicted for $U$. maydis, $U$. hordei, and S. reilianum genes, respectively (e.g. denoted as UMAG_03496: UMAG_03497). Finally, Cufflinks Cuffcompare was utilized to categorize the remaining PASA assemblies. Novel transcript identifiers were created by combining the code provided by Cufflinks Cuffcompare (described in Table 2) with the corresponding MIPS gene identifier. For example, transcript $x 1$ UMAG_02150 and x2-UMAG_02150 represent two unique 


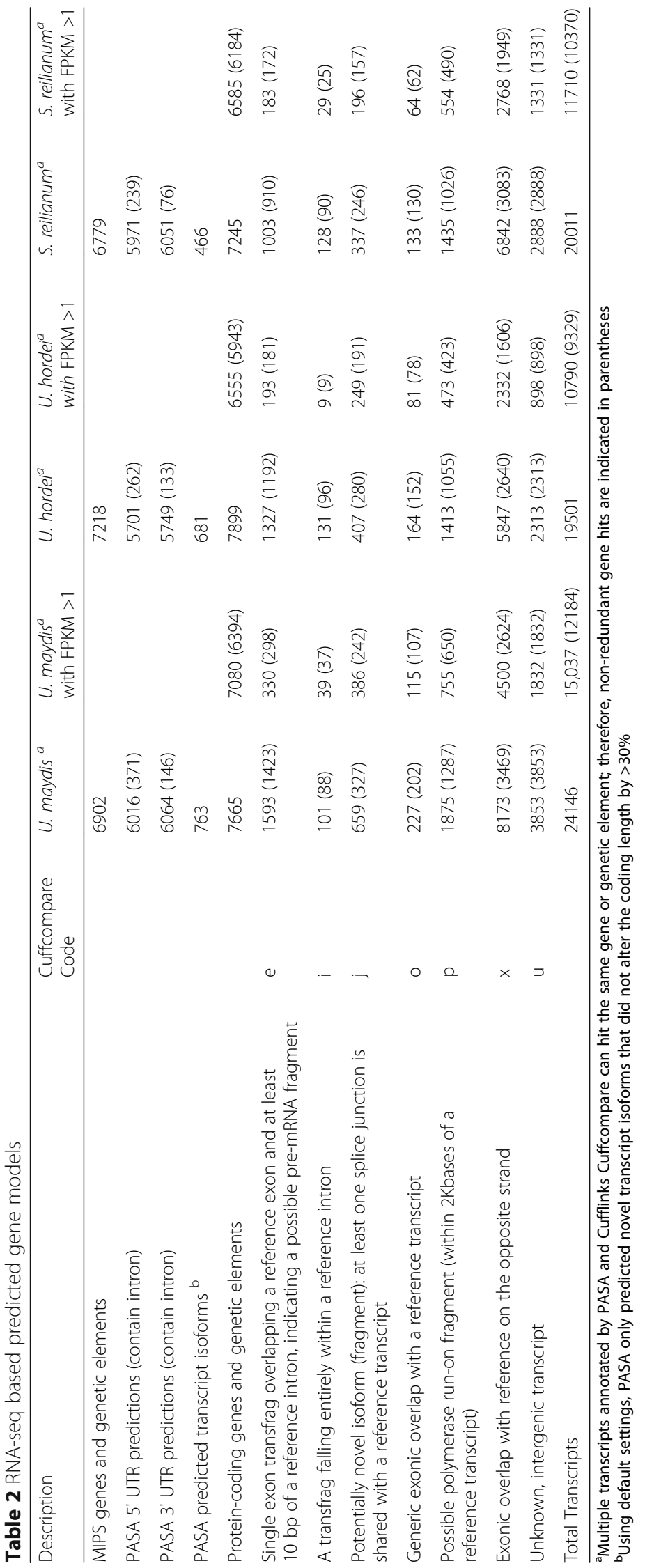


antisense transcripts to the gene UMAG_02150. For the unknown intergenic transcripts (Cuffcompare class ' $\mathrm{u}$ ') $>100 \mathrm{nt}$ in length, identifiers were created by combining "u1-" with the PASA assembly identifier (e.g. u1align_116962). The resulting datasets were used to create updated GFF3-formatted genome annotation files for the three smut fungi (Additional files 3, 4 and 5).

For each RNA-seq library, paired-end reads were mapped to their corresponding genome and updated GFF3 annotation file using CLC Genomics Workbench. Fragments per kilobase of transcript per million mapped reads (FPKM) values were calculated by CLC Genomics Workbench for each transcript. Maximum FPKM values were determined for each transcript across the RNA-seq libraries for $U$. maydis, $U$. hordei, and $S$. reilianum. These maximum FPKM values were then used to estimate the number of detected transcripts using a range of FPKM cut-off values (Additional file 6). Similar to other studies [29-36], a conservative threshold of FPKM >1 was used as the cut-off to indicate whether a transcript was detected by RNA-seq and considered to be expressed by $U$. maydis, $U$. hordei, and S. reilianum cells from which RNA was isolated. If the FPKM value for a given transcript was below 1 , the transcript was considered to be predicted but not detected. Using these designations, RNA-seq-predicted gene models and RNA-seq-detected gene models were determined for $U$. maydis, $U$. hordei, and S. reilianum, respectively (Additional files 7, 8 and 9, summarized in Table 2). For each smut, alternatively spliced isoforms were detected in addition to those predicted by PASA. Overall, this study dramatically increases the number of known transcriptional units for each fungus, as highlighted by the observation that the total number of transcripts detected by RNA-seq (FPKM $>1$ ) in this study were 2.2 times the number of $U$. maydis, 1.5 times the number of $U$. hordei, and 1.7 times the number of $S$. reilianum previously predicted gene models.

\section{Detection of non-coding intergenic transcripts and previ- ously uncharacterized ORFs}

A number of intergenic transcripts (class ' $u$ ' and class 'p') were predicted and detected for each smut. Using these nucleotide sequences, the Trinotate annotation suite predicted full-length ("complete") protein-coding ORFs, and translated those ORFs into peptides for the purposes of identifying similar peptides in known databases using BLASTp, and for locating putative secretion signals using SignalP (Additional files 10, 11 and 12; summarized in Table 3). A small number of intergenic transcripts are predicted to encode proteins and some of these contain secretion signals; however, it is striking that $2414 U$. maydis, 1206 U. hordei, and $1776 \mathrm{~S}$. reilianum transcripts represent novel lincRNAs. Further, RNAmmer analysis of the detected intergenic transcripts identified only one new $S$. reilianum rRNA prediction (u1align_119627). BLASTn comparisons identified lincRNAs with similar sequences among the different smut fungi, indicating some lincRNAs are conserved among the smuts examined in this study (Additional file 13).

\section{Antisense transcription is prevalent and conserved among smut fungi}

Natural antisense transcripts (class ' $x$ ') were detected for 2624 U. maydis, 1606 U. hordei, and 1949 S. reilianum genes. The predicted NATs are presented in Additional files 14, 15 and 16 and the detected NATs in Additional files 17, 18 and 19. Interestingly, antisense transcripts were consistently longer in $U$. hordei (average length of $538 \mathrm{bp}$ ), compared to $U$. maydis (433 bp), and $S$. reilianum (422 bp; Fig. 1). Further, 186, 101, and 34 antisense transcripts were detected that contain introns for $U$. maydis, $U$. hordei, and S. reilianum, respectively (Additional files 7, 8 and 9). There was also cell-type-specific expression of NATs in $U$. maydis. NATs complementary to 1284 genes were detected in the dormant teliospore and 657 of these were not detected in haploids or the dikaryon. Similarly, NATs complementary to 164 genes were detected only in the haploid cells and 164 only in the dikaryon. Comparison analysis identified antisense transcripts that were conserved in two or all three of the smut fungi (Fig. 1). Gene Ontology (GO) term enrichment analyses on the 2617 genes with NATs in $U$. maydis revealed enrichment of the molecular function category binding $(p=0.0405)$ and GO analysis of genes

Table 3 Trinotate results summary for intergenic transcripts

\begin{tabular}{|c|c|c|c|c|c|c|c|}
\hline \multirow[b]{2}{*}{ Fungus } & \multirow[b]{2}{*}{ Transcripts detected } & \multicolumn{3}{|c|}{ Peptides $>30$ aa $<100$ aa } & \multicolumn{3}{|c|}{ Peptides $>100$ aa } \\
\hline & & Peptides $^{a}$ & BLASTp hits & SignalP predictions & Peptides $^{a}$ & BLASTp hits & SignalP predictions \\
\hline U. maydis (class 'u') & 1832 & 113 & 13 & 6 & 28 & 12 & 0 \\
\hline U. maydis (class 'p') & 755 & 21 & 5 & 0 & 11 & 7 & 0 \\
\hline U. hordei (class 'u') & 898 & 58 & 7 & 0 & 26 & 11 & 3 \\
\hline U. hordei (class 'p') & 423 & 29 & 2 & 0 & 2 & 0 & 0 \\
\hline S. reilianum (class 'u') & 1331 & 28 & 0 & 0 & 3 & 1 & 0 \\
\hline S. reilianum (class 'p') & 490 & 10 & 1 & 1 & 4 & 2 & 1 \\
\hline
\end{tabular}

Only peptides with a "complete" open reading frame (methionine-stop codon) and a transcript FPKM > =1 are reported 


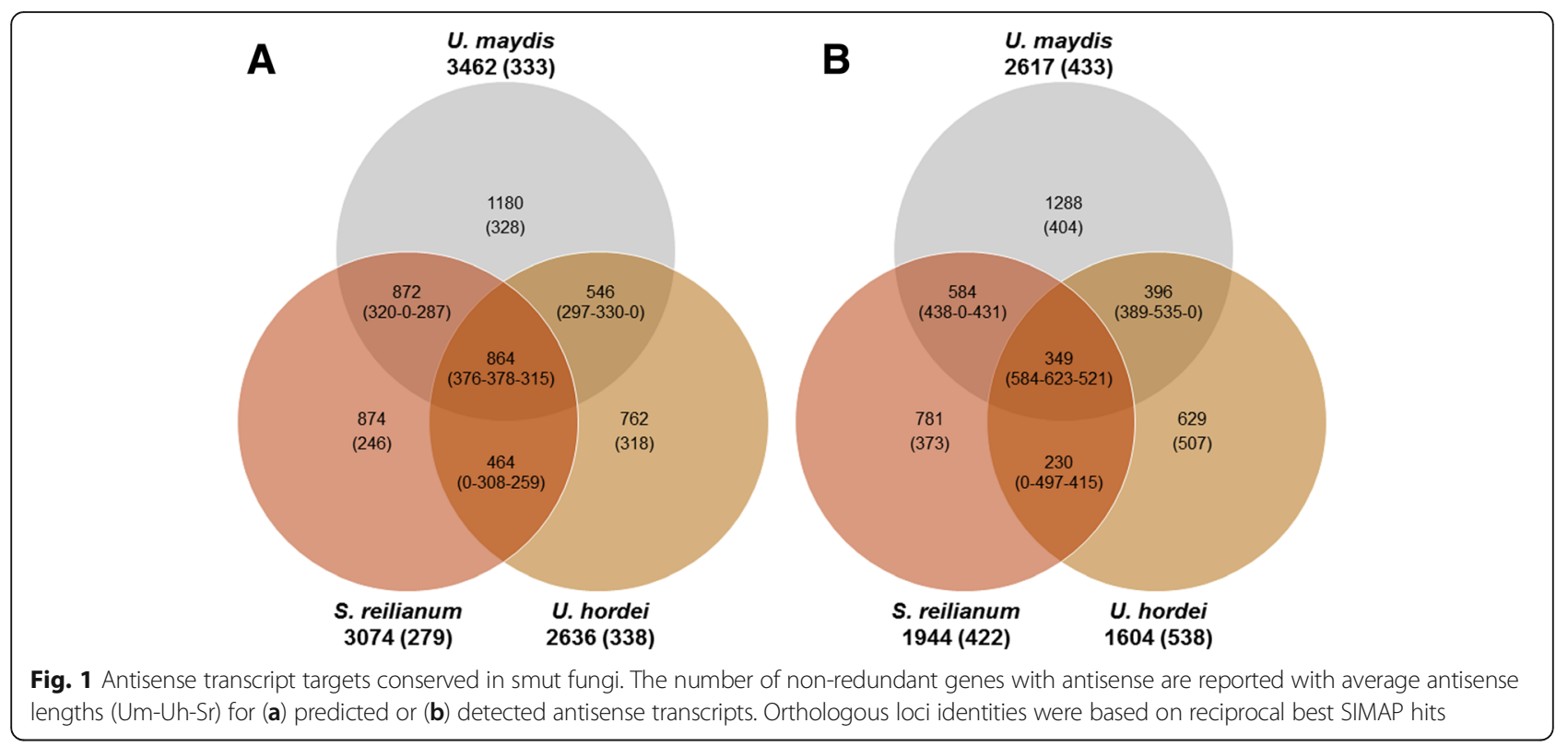

complementary to the 349 NATs conserved among all three smut fungi revealed enrichment in the molecular function categories: binding $(p=0.00128)$, DNA binding $(p=0.0188)$, nucleoside triphosphatase activity $(p=0.0382)$, and its sub-category helicase activity $(p=0.0154)$.

\section{Validation of RNA-seq data}

A number of $U$. maydis transcripts detected by RNA-seq were chosen as candidates for expression pattern confirmation tests. Transcripts with similar annotation features, such as cell-type-specific expression or the presence of an intron, were categorized and independently assessed by RT-PCR in 6 distinct $U$. maydis celltypes. Of the 62 candidate transcripts tested, 61 were detected in at least one cell-type. An intron was detected in at least one cell-type of each candidate antisense transcript tested with predicted introns $(n=5)$. Annotation of transcripts from class 'e,' 'j', and 'o' suggested the presence of two isoforms. Of these transcripts tested $(n=8)$, two isoforms were detected, with the exception of the j1-UMAG_11139 and j2-UMAG_03704 loci where only a single major isoform was found. Examples of transcript confirmations from each category are shown in Fig. 2. Candidate transcripts and overall RT-PCR expression results are summarized in Additional file 20.

\section{Discussion}

In this study, we present deep RNA-seq and genome annotation updates for three closely related smut fungi: $U$. maydis, which causes common smut of corn, $U$. hordei, the causal agent of covered smut of barley, and S. reilianum, which causes head smut of corn. These phytopathogens are threats to cereal crop production worldwide and $U$. maydis has become the model for biotrophic plant pathogenesis. Previous genome annotations for these fungi were based on less comprehensive technologies (e.g. EST analysis) and lacked extensive data on important annotation features such as UTR length, alternative splicing events and non-coding transcription (intergenic and antisense). Our massively parallel stranded RNA-sequencing experiments update and expand $U$. maydis, $U$. hordei, and S. reilianum genome annotations by including data for these features and uncovering conserved NATs and lincRNAs that can influence gene expression on a genome wide level.

\section{Updating protein-coding annotations}

The genome annotations currently available in the MIPS database represent 6902, 7218, and 6779 protein-coding genes in $U$. maydis, $U$. hordei, and $S$. reilianum, respectively. Our analyses permitted us to update thousands of these gene models (Additional files 7, 8 and 9), which included the identification of: transcript isoforms (predicted by PASA to vary by $<30 \%$ from existing gene models), pre-mRNA fragments (Cuffcompare class 'e'), transcripts spanning introns (Cuffcompare class 'i'), alternatively spliced isoforms (Cuffcompare class ' $j$ '), transcripts overlapping exons (Cuffcompare class 'o'), and putative polymerase run-on events (Cuffcompare class ' $p$ '). Table 2 contains a summary of the number of predicted gene models. To confirm our in silico predictions, we screened 62 candidate transcripts in these categories by semi-quantitative RT-PCR. We were able to detect $5 / 5$ pre-mRNA fragments, $3 / 3$ intronic transcripts, $3 / 5$ novel splice isoforms, $5 / 5$ exon overlapping transcripts, and $11 / 12$ putative polymerase run-on transcripts (Additional file 20). The "run-on" transcripts were detected by amplification of an RNA fragment that 


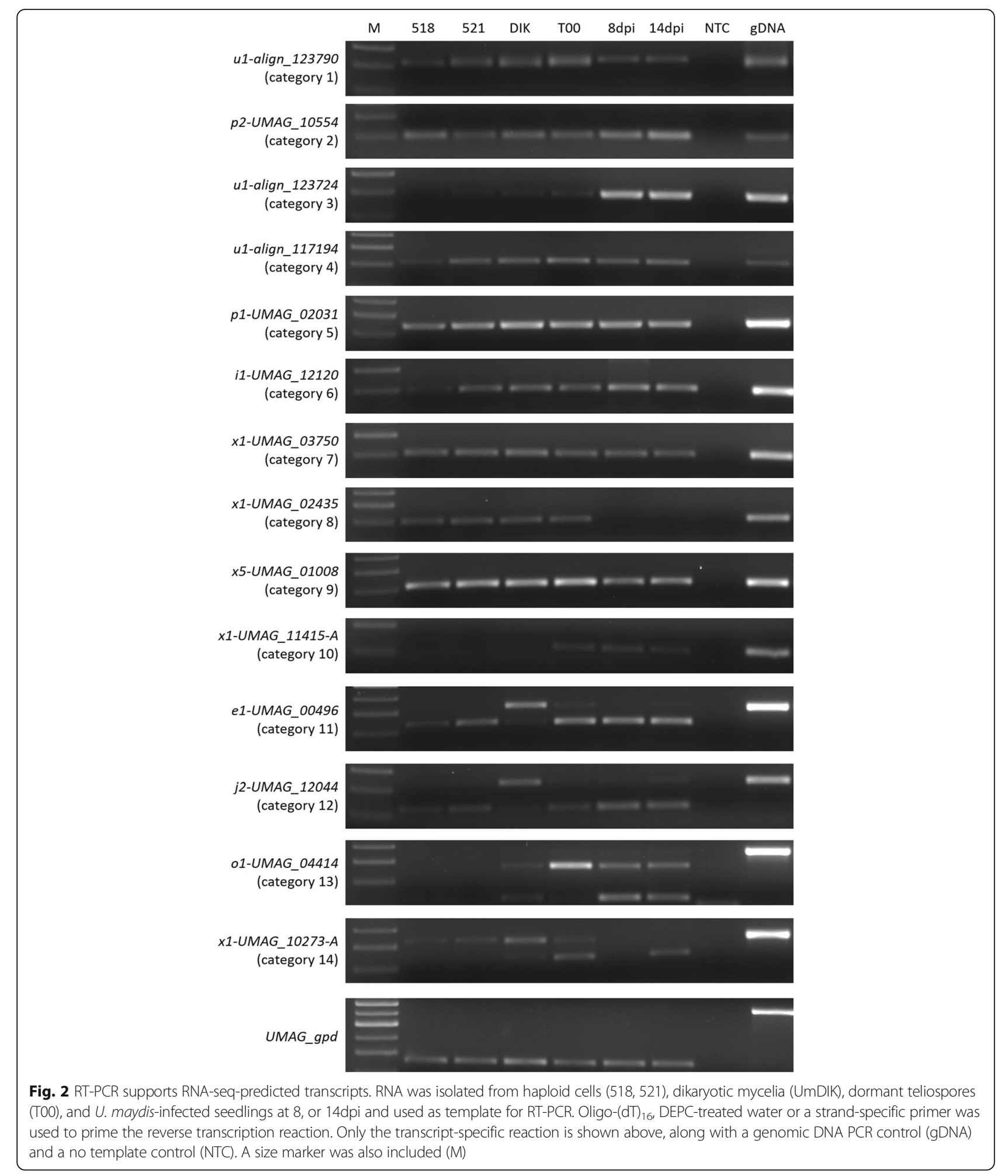

could also represent separate transcripts. Given that the average intergenic lengths are $1127 \mathrm{bp}$ for $U$. maydis, 1186 for $U$. hordei, and 929 bp for S. reilianum [9], the putative polymerase run-on transcripts that are $<2 \mathrm{kbp}$ from a neighbouring gene may actually represent separate transcripts. In addition, we updated UTR annotations for $87 \%$ of $U$. maydis, $79 \%$ of $U$. hordei, and $88 \%$ of S. reilianum MIPS gene models, for which 5' UTRs average $289 \mathrm{bp}, 235 \mathrm{bp}$, and $211 \mathrm{bp}$, and 3' UTRs average $304 \mathrm{bp}, 231 \mathrm{bp}$, and $180 \mathrm{bp}$, respectively. 
The identification of alternative splicing events in all three smuts is noteworthy. Previous research identified significant rates of alternative splicing across the genomes of several ascomycetes and basidiomycetes including $U$. maydis, and suggested that there may be links between alternative splicing and pathogenesis [37]. The rates of alternative splicing they reported were higher in pathogenic fungi $(7.6 \%)$ than non-pathogenic fungi (5.1\%), although not significantly so. Also, the Pfam domain descriptions of genes undergoing alternative splicing in pathogenic fungi showed their involvement in fungal adaptation to the altered host environment, suggesting a potential role for alternative splicing in fungal pathogenesis. The numbers reported here, as well as the results from recent RNA-sequencing of other fungi could expand and update the data used by [37]. For example, Illumina-based RNA-seq has provided the first evidence of alternative splicing in the pathogen F. graminearum, identifying such events at 231 loci [38]. In addition, alternative splicing events were identified at $59 \%$ of Cryptococcus neoformans var. neoformans loci, many of which were altered in response to changes in the environment [39]. We have previously detected alternative splicing events at $3.6 \%$ of $U$. maydis MIPS genes through EST-based analyses [10]. Using the PASApredicted transcript isoforms, our current dataset, conservatively expands this number in $U$. maydis to $\sim 11 \%$, in $U$. hordei to $9 \%$, and in S. reilianum to 7\% (Table 2). These findings open avenues for future research in identifying the role of alternative splicing in smut pathogenesis and the regulation of gene expression as a whole.

In addition to updating current MIPS gene models, our sequencing experiments have greatly expanded the annotation depth for each of the smut genomes. Relative to the original annotations in the MIPS database, the number of detected unique transcripts was increased by 2.2 fold for $U$. maydis, 1.5-fold for $U$. hordei, and 1.7 fold for S. reilianum (Table 2 and Additional files 7, 8 and 9). The larger increase for $U$. maydis was likely due to sequencing RNA from haploids, dikaryons, and teliospores (dormant and germinating) while only RNA from haploids was sequenced for $U$. hordei and only RNA from haploids and dikaryons for S. reilianum. Increased numbers of detected transcripts were also observed by analyses of deep RNA-seq data of $F$. graminearum yielding a 1.2-fold increase and that of Aspergillus nidulans yielding a 4.2-fold increase [38, 40]. Among the novel transcripts detected in our study, a small number have putative ORFs. We identified 173, 115, and 45 putative novel protein-coding genes in $U$. maydis, $U$. hordei, and S. reilianum, respectively. Of these putative ORFs, six $U$. maydis, three $U$. hordei, and two $S$. reilianum peptides contain a putative secretion signal as determined by SignalP analyses, suggesting these novel ORFs could correspond to effector proteins (Additional files 10, 11 and 12). In identifying potential effector proteins, it is critical to correctly delineate 5'UTRs. The 5' UTR data presented here is consistent with earlier 5' UTR determinations from full-length cDNA analyses [14]; however, it is possible that the data set is still not comprehensive. If this was the case then some starts of transcription may have been missed and this would mean that some effector genes may not have been recognized. As such, the additional effector candidate genes presented here must be considered a minimum number. Even if this was the case, it would not substantially increase the number of predicted proteins or effectors. Other comparable transcriptome studies include updating the genome annotation of $A$. nidulans, in which $29 \%$ of the reads mapped to unannotated loci, and of these $\sim 13,000$ transcripts, 343 were predicted to code functional proteins based on Pfam/Rfam analyses. This corresponded to a proteincoding gene annotation increase of $\sim 2.9 \%$ [40]. Similarly, our expansion of the $U$. maydis protein-coding annotations corresponds to an increase of $2.5 \%$, while $U$. hordei and $S$. reilianum additions correspond to increases of $1.6 \%$ and $0.6 \%$, respectively (Additional files 10, 11 and 12). The lower numbers for the latter two smuts are likely due to sequencing fewer libraries relative to $U$. maydis. With more comprehensive RNA-seq for these smuts, the number of updated protein-coding annotations and the number of newly identified transcripts would likely increase further.

\section{Expansion of the non-coding transcriptome}

Although only a small number of the thousands of novel transcripts we detected potentially encode functional proteins, the vast majority do not. These transcripts are, therefore, considered ncRNAs. There is increasing evidence that ncRNAs are functional and there is support for their influence on the expression of other genes. Their functions have been linked to key cellular functions, cancer and even aging $[15,41]$. The large numbers of ncRNAs identified here in the smut fungi fall into two categories: lincRNAs and NATs.

\section{Long intergenic non-coding RNAs (lincRNAs)}

Through RNA-seq analyses, 2414 lincRNAs $\geq 100 \mathrm{nt}$ were identified in $U$. maydis, 1206 in $U$. hordei, and 1776 in S. reilianum (Table 3). All $U$. maydis lincRNAs that were screened for using RT-PCR were detected (13/ 13; Additional file 20), providing confidence in their existence. These lincRNA numbers are an order of magnitude higher than those identified by RNA-seq studies in other filamentous fungi. Large-scale RNA-seq analyses detected 462 lincRNAs in Neurospora crassa [25], 121 lincRNAs in F. graminearum [4], 120 lincRNAs in C. graminicola [3], and 155 lincRNAs in $R$. solani 
[21]. Although these studies detected wide-spread transcription of lincRNAs in filamentous fungi, there has been limited functional analysis conducted. In $C$. neoformans, the lincRNA RZE1 controls yeast-to-hypha morphological transition by regulating a zinc finger transcription factor Znf2 [42] and in $U$. maydis, a lincRNA (ncRNA1) influences level of virulence [11], though the mechanism by which ncRNA1 functions is not yet known. Taken together, these studies indicate that lincRNA transcription is pervasive among the filamentous fungi with the number of lincRNAs substantially higher in the smut fungi. A comprehensive detection of possible lincRNA orthologs among the smut fungi would require complete genome assemblies to enable accurate positional identification of the lincRNAs. This is not currently the case, so our searches were restricted to assessing nucleotide sequence similarity. Among the thousands of $U$. maydis, $U$. hordei, and S. reilianum lincRNAs identified here, there was sufficient sequence level conservation to identify 34 potential lincRNA orthologs present in two or all three of the smut fungi. The identification of lincRNA orthologs supports the hypothesis that some of these have functions in the smut fungi.

\section{Natural antisense transcripts (NATs)}

This report is the first to utilize strand-specific RNA-seq to investigate transcriptomes of smut fungi. The strandspecificity enabled large-scale detection of NATs. Antisense transcription was predicted/detected at 50\%/38\% of the $U$. maydis loci, $36 \% / 22 \%$ of the $U$. hordei loci, and $45 \% / 29 \%$ of the S. reilianum loci (Table 2). These percentages of protein-coding loci with antisense transcripts are higher than those reported for other filamentous fungi. A recent deep analysis of the $A$. nidulans transcriptome identified at least one antisense read mapped to $72 \%$ of annotated loci, however, this was decreased to $14 \%$ when only those transcripts with a RPKM values $>1$ were considered [40] and, in $N$. crassa, antisense transcripts were present at only $\sim 5 \%$ of the annotated protein-coding loci [25]. The increased percentage of loci with NATs in $U$. maydis relative to the other smuts likely reflects differences in sequence depth; however, it is noteworthy that $U$. maydis lacks a functional RNAi pathway while $U$. hordei and S. reilianum have retained the RNAi machinery $[9,24]$. Given that fungal NATs have been shown to undergo cleavage by Dicer (e.g. [25]), we expected that NATs in $U$. hordei and S. reilianum would be smaller, on average, due to the presence of functional RNAi machinery. However, the average length of $S$. reilianum NATs (422 bp) was comparable to average $U$. maydis NATs (433 bp), and $U$. hordei NATs were longer on average (538 bp) (Additional files 17, 18 and 19). These findings suggested that if the detected
NATs are functional in these species, they likely exert their functions without involvement of an RNAi pathway.

The functional characterization of NATs in filamentous fungi has identified roles in development, metabolism, and pathogenesis (Reviewed in [18]). In the mushroom-forming basidiomycete Coprinopsis cinerea, a large number of antisense transcripts were identified, and a substantial subset of these NATs have expression patterns consistent with their having a role in fruiting body development, although clear functional analysis remains to be carried out [16]. Roles for NATs in regulating cellular metabolism stem from studies in $N$. crassa and $U$. maydis. In $N$. crassa, light-sensitive NATs regulate the circadian rhythm $[43,44]$. The core oscillator gene frequency ( $r q$ ) and a long-non-coding antisense to frq, qrf, regulate each other's expression through transcriptional interference in a negative feedback loop that involves chromatin modifications and premature transcriptional termination [43]. In $U$. maydis, a teliosporespecific NAT (as-ssm1) to the mitochondrial seryl-tRNA synthetase (ssm1) regulates the stability of $\operatorname{ssm} 1 \mathrm{mRNA}$ through its direct binding, and also influences growth rate, mitochondrial membrane potential, and oxygen consumption rates [19]. These data led to the presentation of a model in which as-ssm1 expression in the dormant teliospore (1) contributes to the suppression of mitochondrial function during dormancy through direct binding of $\operatorname{ssm} 1$ and (2) preserves $\operatorname{ssm} 1$ mRNAs for rapid translation upon germination. An indication of a role for NATs in regulating pathogenesis comes from investigating the bidirectional regulatory region that controls the expression of teliospore-specific NATs to a $U$. maydis xylitol dehydrogenase (UMAG_02150) and a D-gluconic acid reductase (UMAG_02151) gene, as well as $n c R N A 1[11,15]$. Deletion of this regulatory region altered the expression of the NATs and ncRNA1 and significantly reduced pathogenesis. This indicated that proper control of NAT expression is required for full pathogenesis. The details of how the altered regulation impacts pathogenesis are under investigation (Goulet et al., unpublished). It is likely that there are other functional antisense present in the teliospore. RNA-seq detected 116 teliospore-specific NATs, 164 haploid cellspecific NATs, and 164 dikaryon-specific NATs. Confirming cell-type-specific expression of these NATs will identify candidates for future functional investigation.

Given the widespread existence of NATs in filamentous fungi and their emerging functional relevance [18, $45,46]$, it is anticipated that a large number of the NATs we have detected in this study are functional. Interestingly, $\sim 50 \%$ of the $U$. maydis NATs are conserved in either $U$. hordei ( 15\%), S. reilianum $(\sim 22 \%)$ or both $(\sim 13 \%)$ (Fig. 1). In vitro analyses confirmed the presence 
of 18/18 U. maydis NATs that were screened, several of which were predicted to contain introns, overlap known effector proteins or be expressed in a cell-type-specific manner. Conservation between species and expression in specific cell-types are characteristics which point to functional relevance [47]. The identification of such a high proportion (nearly one fifth) of genes with conserved NATs suggests their potential involvement in something of broad influence within the smut fungi. As an initial functional characterization, we conducted GO term enrichment analyses on the NATs conserved among the smut fungi. The analyses revealed that the NATs conserved among all three smut fungi are complementary to genes that are enriched in the molecular function categories: binding, DNA binding, nucleoside triphosphatase activity, and its sub-category helicase activity. This indicates that genes in these categories in the smut fungi have homologous complementary antisense transcripts. If the presence of these NATs is independently confirmed in all species, it could indicate a common mechanism of modulating the expression of these genes. Since the genes in the DNA binding and helicase activity categories alone include histones, transcription factors, chromatin modification enzymes, proteins involved in DNA replication including polymerase subunits, as well as DNA and RNA helicases, this enriched group of genes have the potential to influence gene expression at multiple levels. Therefore, if the expression of these genes is modulated by antisense, then antisense could influence multiple levels of gene expression control and through this, practically all aspects of fungal development. Further, as stated earlier, these conserved NATs must be functioning in an RNAiindependent manner. Interestingly, the $\mathrm{GO}$ analysis also provides a basis for a hypothesis regarding how this might occur. Our initial hypotheses are that 1) NATs influence the expression of genes that can modulate gene expression and 2) this NAT influence is modulated through a feedback mechanism involving NAT control of protein expression and protein control of NAT expression. Components of these hypotheses are currently being investigated using NATs and helicases preferentially expressed in the teliospore. As such, this RNA-seq analysis has substantially advanced the knowledge of a model organism and provided the basis for investigating NAT-mediated regulation as a new level of gene expression control in phytopathogenic fungi.

\section{Conclusions}

In the present study, we have expanded and updated the genome annotations for three closely related smut fungi, $U$. maydis, $U$. hordei, and S. reilianum. We have updated incomplete or incorrect MIPS gene models, and have added information regarding UTR lengths and alternative splicing. Hundreds of novel putative protein-coding genes have been identified across the three genomes, a handful of which may represent effector proteins. Finally, our analyses enabled the detection of thousands of lincRNAs and NATs in these smuts, and the determination that a high proportion of the NATs are conserved among the three fungi. This provides the basis of hypotheses regarding NAT function and a starting point for functional analyses, which may reveal important roles for ncRNAs in these species.

\section{Methods}

\section{Fungal strains, growth conditions and cultivation of cell-types}

All fungal strains used in this study are listed in Table 1. $U$. maydis and S. reilianum cultures were incubated at $28{ }^{\circ} \mathrm{C}$ and $U$. hordei cultures were incubated at $22{ }^{\circ} \mathrm{C}$, unless otherwise noted. Haploid $U$. maydis strains 518 and 521, $U$. hordei strains Uh364 and Uh365, and S. reilianum strains SRZ1 and SRZ2 were inoculated from frozen stocks onto potato dextrose agar (PDA) plates. Single colonies from each plate were incubated in $3 \mathrm{~mL}$ potato dextrose broth (PDB) shaking at $250 \mathrm{rpm}$. After $\sim 48 \mathrm{~h}$, cellular concentrations were normalized to an $\mathrm{OD}_{600}$ of 1.0, and $10 \mu \mathrm{L}$ of each haploid strain was spotted on PDA plates supplemented with $2 \%$ activated charcoal (10 spots per plate, 8 plates per strain), and incubated at room temperature (RT). $518 \times 521$ and SRZ1 $\times$ SRZ2 forced dikaryon growth was induced under similar conditions, as described in [11]. Briefly, haploid cultures were grown overnight as described above and diluted with sterile water to an $\mathrm{OD}_{600}$ of 1.5. Equal volumes of compatible haploid cultures were combined in a $1.5 \mathrm{~mL}$ tube so that $10 \mu \mathrm{L}$ of each mixture could be spotted on PDA plates supplemented with $2 \%$ activated charcoal (10 spots per plate, 8 plates per strain). After 2 days of growth, haploid and dikaryon colonies were inspected visually for the presence or absence of filamentous growth. Colony morphology was confirmed and documented using a Leica S8 APO stereo microscope (Leica Microsystems), as described in [48]. Haploid or dikaryon cells were scraped from the medium with a scoopula, immediately frozen in liquid nitrogen, and stored at $-80{ }^{\circ} \mathrm{C}$ until RNA isolation. $U$. maydis $518 \times 521$ teliospores were harvested from mature tumours of corn (Z. mays L. 'Golden Bantam') as described in [49] with the following changes: overnight haploid cell cultures were diluted with sterile water to an $\mathrm{OD}_{600}$ of 1.0, equal volumes of compatible haploids were combined in $50 \mathrm{~mL}$ conical tubes, and $6 \mathrm{~mL}$ of this mixture was injected down the cob silk shaft. Harvested teliospores were germinated in $\mathrm{PDB}+$ Streptomycin $(160 \mu \mathrm{g} / \mathrm{mL})$ shaking $90 \mathrm{rpm}$. Teliospores were sampled at $0 \mathrm{~h}, 9 \mathrm{~h}$, and $18 \mathrm{~h}$ post induction of germination 
(PIG). Teliospore germination was observed at 400X magnification using a Axio Scope.A1 compound microscope (Carl Zeiss MicroImaging), as described in [48]. For RT-PCR experiments, in addition to the cell-types described above, 7-day-old seedlings were infected with $518 \times 521$, as described in [1]. U. maydis-infected plant material was harvested at 8 and 14 days post infection (DPI), immediately frozen in liquid nitrogen, and stored at $-80{ }^{\circ} \mathrm{C}$ until RNA isolation.

\section{RNA extraction, CDNA library preparation and RNA- sequencing}

Total RNA was isolated, precipitated, and DNase I-treated following methods outlined in [11]. Briefly, harvested cells were ground in liquid nitrogen using a mortar and pestle. The ground cells or teliospores were resuspended in TRIzol reagent (Life Technologies) and transferred to $2 \mathrm{~mL}$ screw-cap tubes containing Lysing Matrix C (MP Biomedicals). Cells were disrupted as described by [49] using a bead-mill and RNA was isolated following the manufacturer's protocol. RNA was treated with DNAseI (New England Biolabs) to remove contaminating DNA. RNA was precipitated using RNA precipitation solution and isopropanol [50], washed with $75 \%$ ethanol, and resuspended in nuclease-free water (Life Technologies). RNA quality was confirmed through electrophoresis of glyoxalated RNA on a denaturing agarose gel (1X BPTE) as outlined in [50]. Total RNA was sent to the Clinical Genomics Centre at Mount Sinai Hospital (Toronto, Canada). RNA quality was assessed using a Bioanalyzer (Agilent Technologies) and all samples had an RNA integrity number (RIN) greater than 7.5. Poly(A) mRNA was enriched using oligo dT-beads and cDNA libraries were prepared using the TruSeq Stranded mRNA Library Preparation kit (Illumina Inc.). Barcoded libraries were pooled in equimolar quantities and two libraries were sequenced per lane on a HiSeq 2500 System (Illumina Inc.) to generate 75 bp pairedend reads.

\section{RNA-sequencing read alignment, transcript characterization, and gene ontology enrichment}

For each fungal species, the resulting sequences were pooled and then assembled into de novo transfrags using Trinity [26] with the following settings: --SS_lib_type RF, --min_kmer_cov $=2$, --min_contig_length $=100,-$-normalize_reads, and -jaccard_clip. Genome-guided Trinity transfrags were also predicted with the following additional setting: --genome_guided_max_intron $=1500$. The published genome annotations $[1,8,9]$ were used as references by the program to assemble spliced alignments (PASA; [27]), which created updated gene models for each fungus using the de novo and genome-guided transfrag assemblies. These predicted gene models were then categorized with Cufflinks Cuffcompare [28]. For each library, paired-end reads were aligned to their respective genome sequence using CLC Genomics Workbench v9.0 (Qiagen) with the following changes to default settings: mapping type = map to gene regions only, strand specific $=$ reverse, count paired reads as two $=$ no, expression value $=$ FPKM . This resulted in the calculation of fragments per kilobase of transcript per million mapped reads (FPKM) values by CLC Genomics Workbench for each transcript. Transcripts were considered to be specifically expressed in a cell-type if they had an FPKM $>=1$ in one cell-type and $<1$ in others. For haploid cells FPKM $>=1$ were required in both the 518 and 521 libraries. For dormant teliospores, FPKM $>=1$ was required for the T00 library (and not the T09 or T18 germinating teliospore libraries). We used portions of the Trinotate annotation suite (https://trinotate.github.io/) to further characterize novel transfrags, including: BLASTp sequence similarity search to known sequence data (SwissProt database, August 2014), RNAmmer v1.2 [51], and protein signal peptide prediction (SignalP v4.0) [52]. Similarity Matrix of Proteins (SIMAP) values have previously been used to identify paralogs between smut species [9], and using a smaller dataset, [53] identified putative fungal orthologs using a reciprocal best-BLASTp hit approach. In this study, the two methods were combined and orthologs were identified as reciprocal best SIMAP hits. When calculating the number of orthologous antisense in Fig. 1, the merged transcripts were excluded because they could be Trinity-related artifacts resulting from the compact fungal genomes that may contain overlapping UTRs. For $U$. maydis genes, gene ontology term enrichment [54] was assessed using the web-based g:Profiler [55] with a g: SCS significance threshold $<0.05$. To identify lincRNAs with similar sequences among the smut fungi, in-house BLAST databases were created from the updated gene models described above; therefore, these databases contained both coding and non-coding nucleotide sequences. BLAST 2.5.0+ [56] was performed with an e-value cutoff of 1E-03 to identify class 'p' and class ' $\mathrm{u}$ ' transcripts with similar sequence composition in the other two smuts.

\section{Transcript expression analysis}

Genome sequences from the MIPS $U$. maydis database (MUMDB; [57]) were used to design strand-specific first-strand synthesis primers, as well as PCR primers, as described in $[15,58]$. All primers used in this study are listed in Additional file 21. Antisense strand-specific first-strand synthesis primers were designed based on RNA-seq-predicted transcript structures. First-strand synthesis primers included oligo $(\mathrm{dT})_{16}$, DEPC-treated $\mathrm{H}_{2} \mathrm{O}$, a sense-specific first-strand primer or an antisensespecific first-strand primer. First strand synthesis was conducted on 200 ng of DNase I-treated RNA, using the 
TaqMan Gold RT-PCR kit (Applied Biosystems) following methods outlined in [58]. cDNA was diluted eightfold (1:7) with $\mathrm{dH}_{2} \mathrm{O}$. PCRs were conducted using the AmpliTaq Gold DNA Polymerase Kit (Applied Biosystems) following the manufacturer's recommended protocol. One third of the PCR products were visualized using agarose gel electrophoresis (1X TAE) and were subsequently stained with $0.3 \mu \mathrm{g} / \mathrm{mL}$ ethidium bromide (BioShop).

\section{Additional files}

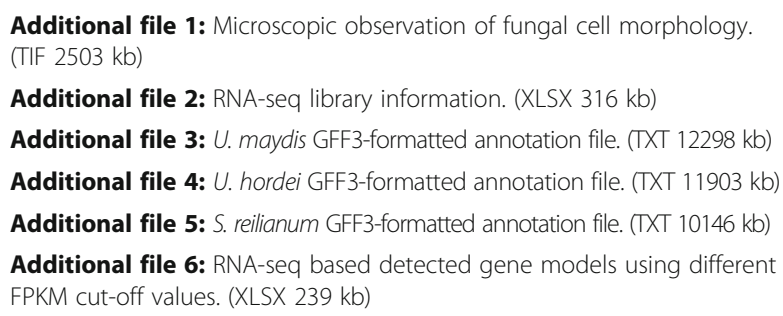

Additional file 7: U. maydis RNA-seq based predicted gene models. (XLSX $661 \mathrm{~kb}$ )

Additional file 8: $U$. hordei RNA-seq based predicted gene models. (XLSX $644 \mathrm{~kb}$ )

Additional file 9: $S$. reilianum RNA-seq based predicted gene models. (XLSX $644 \mathrm{~kb})$

Additional file 10: Trinotate results for $U$. maydis intergenic transcripts. (XLSX $251 \mathrm{~kb}$ )

Additional file 11: Trinotate results for $U$. hordei intergenic transcripts. (XLSX $247 \mathrm{~kb}$ )

Additional file 12: Trinotate results for S. reilianum intergenic transcripts. (XLSX $242 \mathrm{~kb}$ )

Additional file 13: BLASTn results find intergenic transcripts with similar sequences in smuts. (XLSX $285 \mathrm{~kb}$ )

Additional file 14: $U$. maydis predicted antisense and putative orthologs with predicted antisense. (XLSX $436 \mathrm{~kb}$ )

Additional file 15: $U$. hordei predicted antisense and putative orthologs with predicted antisense. (XLSX $392 \mathrm{~kb}$ )

Additional file 16: $S$. reilianum predicted antisense and putative orthologs with predicted antisense. (XLSX 417 kb)

Additional file 17: $U$. maydis detected antisense and putative orthologs with detected antisense. (XLSX $386 \mathrm{~kb}$ )

Additional file 18: $U$. hordei detected antisense and putative orthologs with detected antisense. (XLSX $332 \mathrm{~kb}$ )

Additional file 19: $S$. reilianum detected antisense and putative orthologs with detected antisense. (XLSX $351 \mathrm{~kb}$ )

Additional file 20: RT-PCR results supporting in silico analysis. (XLSX $242 \mathrm{~kb}$ )

Additional file 21: Primers used in this study. (XLSX $244 \mathrm{~kb}$ )

\section{Abbreviations}

DPI: Days post infection; EST: Expressed-sequence tag; FPKM: Fragments pe kilobase of transcript per million mapped reads; lincRNA: Long intergenic non-coding RNA; NAT: Natural antisense transcript; ncRNA: Non-coding RNA; PIG: Post induction of germination; RIN: RNA integrity number; RPKM: RPKM Reads per kilobase of transcript per million mapped reads; RT: Room temperature; UTR: Untranslated region

\section{Acknowledgements}

We thank Amanda Seto at Trent University for technical assistance with the teliospore germination assays.

\section{Funding}

We acknowledge funding provided by NSERC of Canada and the Ministry of Ontario Research and Innovation, Ontario Research Fund Research Excellence award program to BJS. We also acknowledge funding through Ontario Graduate Scholarships for LAO and KMG as well as a NSERC of Canada scholarship for KMG.

\section{Availability of data and materials}

All RNA-seq fastq files are available from the NCBI Sequence Read Archive database (accession number SRP099003). All other data is contained in the manuscript and in the supporting material.

\section{Authors' contributions}

MED contributed to the conception of the study, collected and isolated RNA samples, performed RNA-seq data analysis, and drafted portions of the manuscript. LAO drafted portions of the manuscript and, with KMG, conducted RT-PCRs. BJS conceived of and coordinated the project and edited the manuscript. All authors read and approved the final manuscript.

\section{Competing interests}

The authors declare that they have no competing interests.

\section{Consent for publication}

Not Applicable.

\section{Ethics approval and consent to participate}

The fungal strains used in this study were lab strains used with permission from the individuals who supplied them. The Ustilago maydis strains were obtained from Sally Leong University of Wisconsin, Madison; The Ustilago hordei strains were obtained from Guus Bakkeren, Agriculture and Agri-food Canada (AAFC), Summerland; and the Sporisorium reilianum strains were obtained from Jan Schirawski, Georg-August-Universität Göttingen. The plant species used was Zea mays L. 'Golden Bantam' obtained from Ontario Seed Company, Waterloo, ON Canada. Infections were carried out in a greenhouse in compliance with Trent University biosafety guidelines.

\section{Publisher's Note}

Springer Nature remains neutral with regard to jurisdictional claims in published maps and institutional affiliations.

\section{Author details}

${ }^{1}$ Environmental and Life Sciences Graduate Program, Trent University, Peterborough K9L 0G2, ON, Canada. ${ }^{2}$ Forensic Science Program, Trent University, Peterborough K9L 0G2, ON, Canada. ${ }^{3}$ Present Address: Department of Laboratory Medicine and Pathobiology, Faculty of Medicine, University of Toronto, Toronto M5S 1A8, ON, Canada.

Received: 18 January 2017 Accepted: 25 April 2017

Published online: 02 May 2017

\section{References}

1. Kamper J, Kahmann R, Bolker M, Ma LJ, Brefort T, Saville BJ, et al. Insights from the genome of the biotrophic fungal plant pathogen Ustilago maydis. Nature 2006:444:97-101.

2. Van Kan JAL, Stassen JHM, Mosbach A, Van Der Lee TAJ, Faino L, Farmer $A D$, et al. A gapless genome sequence of the fungus Botrytis cinerea. Mol Plant Pathol. 2016;18:75-89.

3. Schliebner I, Becher R, Hempel M, Deising HB, Horbach R. New gene models and alternative splicing in the maize pathogen Colletotrichum graminicola revealed by RNA-Seq analysis. BMC Genomics. 2014;15:1-13.

4. King R, Urban M, Hammond-Kosack MCU, Hassani-Pak K, Hammond-Kosack KE. The completed genome sequence of the pathogenic ascomycete fungus Fusarium graminearum. BMC Genomics. 2015;16:1-21.

5. Tanaka S, Brefort T, Neidig N, Djamei A, Kahnt J, Vermerris W, et al. A secreted Ustilago maydis effector promotes virulence by targeting anthocyanin biosynthesis in maize. Elife. 2014;3:e01355

6. Doehlemann G, Van Der Linde K, Aßmann D, Schwammbach D, Hof A Mohanty A, et al. Pep1, a secreted effector protein of Ustilago maydis, is required for successful invasion of plant cells. Plos Pathog. 2009:5(2): e1000290. 
7. Brefort T, Tanaka S, Neidig N, Doehlemann G, Vincon V, Kahmann R. Characterization of the largest effector gene cluster of Ustilago maydis. Plos Pathog. 2014;10(7):e1003866.

8. Schirawski J, Mannhaupt G, Munch K, Brefort T, Schipper K, Doehlemann G, et al. Pathogenicity determinants in smut fungi revealed by genome comparison. Science. 2010;330:1546-8.

9. Laurie JD, Ali S, Linning R, Mannhaupt G, Wong P, Guldener U, et al. Genome comparison of barley and maize smut fungi reveals targeted loss of RNA silencing components and species-specific presence of transposable elements. Plant Cell. 2012;24:1733-45.

10. Ho EC, Cahill MJ, Saville BJ. Gene discovery and transcript analyses in the corn smut pathogen Ustilago maydis: expressed sequence tag and genome sequence comparison. BMC Genomics. 2007:8:334.

11. Morrison EN, Donaldson ME, Saville BJ. Identification and analysis of genes expressed in the Ustilago maydis dikaryon: uncovering a novel class of pathogenesis genes. Can J Plant Pathol. 2012;34:417-35.

12. Sacadura NT, Saville BJ. Gene expression and EST analyses of Ustilago maydis germinating teliospores. Fungal Genet Biol. 2003;40:47-64.

13. Nugent KG, Choffe K, Saville BJ. Gene expression during Ustilago maydis diploid filamentous growth: EST library creation and analyses. Fungal Genet Biol. 2004;41:349-60.

14. Doyle CE, Donaldson ME, Morrison EN, Saville BJ. Ustilago maydis transcript features identified through full-length cDNA analysis. Mol Genet Genomics. 2011;286:143-59

15. Donaldson ME, Saville BJ. Ustilago maydis natural antisense transcript expression alters mRNA stability and pathogenesis. Mol Microbiol. 2013;89:29-51

16. Muraguchi H, Umezawa K, Niikura M, Yoshida M, Kozaki T, Ishii K, et al. Strand-specific RNA-seq analyses of fruiting body development in Coprinopsis cinerea. Plos One. 2015;10:e0141586.

17. Chacko N, Lin X. Non-coding RNAs in the development and pathogenesis of eukaryotic microbes. Appl Microbiol Biotechnol. 2013;97:7989-97.

18. Donaldson ME, Saville BJ. Natural antisense transcripts in fungi. Mol Microbiol. 2012;85:405-17.

19. Ostrowski LA, Saville BJ. Natural antisense transcripts to nucleus-encoded mitochondrial genes are linked to Ustilago maydis teliospore dormancy. Mol Microbiol. 2016; doi: 10.1111/mmi.13587.

20. Mueth NA, Ramachandran SR, Hulbert SH. Small RNAs from the wheat stripe rust fungus (Puccinia striiformis f.sp. tritici). BMC Genomics. 2015;16:1-16.

21. Hane JK, Anderson JP, Williams AH, Sperschneider J, Singh KB. Genome sequencing and comparative genomics of the broad host-range pathogen Rhizoctonia solani AG8. Plos Genet. 2014;10:e1004281.

22. Kellner R, Bhattacharyya A, Poppe S, Hsu TY, Brem RB, Stukenbrock EH. Expression profiling of the wheat pathogen Zymoseptoria tritici reveals genomic patterns of transcription and host-specific regulatory programs. Genome Biol Evol. 2014:6:1353-65.

23. Christensen JJ. Corn smut caused by Ustilago maydis. Am Phytophatological Soc. 1963:4:129-88.

24. Laurie JD, Linning R, Bakkeren G. Hallmarks of RNA silencing are found in the smut fungus Ustilago hordei but not in its close relative Ustilago maydis. Curr Genet. 2008;53:49-58.

25. Arthanari Y, Heintzen C, Griffiths-Jones S, Crosthwaite SK. Natural antisense transcripts and long non-coding RNA in Neurospora crassa. Plos One. 2014;9:e91353.

26. Grabherr MG, Haas BJ, Yassour M, Levin JZ, Thompson DA, Amit I, et al. Fulllength transcriptome assembly from RNA-Seq data without a reference genome. Nat Biotechnol. 2011;29:644-52.

27. Haas BJ, Delcher AL, Mount SM, Wortman JR, Smith RK, Hannick LI, et al. Improving the Arabidopsis genome annotation using maximal transcript alignment assemblies. Nucleic Acids Res. 2003;31:5654-66.

28. Trapnell C, Williams BA, Pertea G, Mortazavi A, Kwan G, van Baren MJ, et al. Transcript assembly and quantification by RNA-Seq reveals unannotated transcripts and isoform switching during cell differentiation. Nat Biotechnol. 2010;5:511-5.

29. Ramsköld D, Wang ET, Burge CB, Sandberg R. An Abundance of ubiquitously expressed genes revealed by tissue transcriptome sequence data. Plos Comput Biol. 2009;5:e1000598.

30. Brooks MJ, Rajasimha HK, Roger JE, Swaroop A. Next-generation sequencing facilitates quantitative analysis of wild-type and $\mathrm{Nrl}(-/-)$ retinal transcriptomes. Mol Vis. 2011;17:3034-54.
31. Hebenstreit D, Fang M, Gu M, Charoensawan V, van Oudenaarden A, Teichmann SA. RNA sequencing reveals two major classes of gene expression levels in metazoan cells. Mol Syst Biol. 2011;7:497.

32. Vogel C, Marcotte EM. Insights into the regulation of protein abundance from proteomic and transcriptomic analyses. Nat Rev Genet. 2012;13:227-32.

33. Fagerberg L, Oksvold P, Skogs M, Älgenäs C, Lundberg E, Pontén F, et al. Contribution of antibody-based protein profiling to the human chromosome-centric proteome project (C-HPP). J Proteome Res. 2013;12:2439-48.

34. Gonzàlez-Porta M, Frankish A, Rung J, Harrow J, Brazma A. Transcriptome analysis of human tissues and cell lines reveals one dominant transcript per gene. Genome Biol. 2013;14:1-11.

35. Chettoor AM, Givan SA, Cole RA, Coker CT, Unger-Wallace E, Vejlupkova Z, et al. Discovery of novel transcripts and gametophytic functions via RNA-seq analysis of maize gametophytic transcriptomes. Genome Biol. 2014;15:1-23.

36. Sun X, Yang Q, Deng Z, Ye X. Digital inventory of Arabidopsis transcripts revealed by 61 RNA sequencing samples. Plant Physiol. 2014;166:869-78.

37. Grützmann K, Szafranski K, Pohl M, Voigt K, Petzold A, Schuster S. Fungal alternative splicing is associated with multicellular complexity and virulence: a genome-wide multi-species study. DNA Res. 2014;21:27-39.

38. Zhao C, Waalwijk C, de Wit PJGM, Tang D, van der Lee T. RNA-Seq analysis reveals new gene models and alternative splicing in the fungal pathogen Fusarium graminearum. BMC Genomics. 2013;14:21.

39. Gonzalez-Hilarion S, Paulet D, Lee K-T, Hon C-C, Lechat P, Mogensen E, et al. Intron retention-dependent gene regulation in Cryptococcus neoformans. Sci Rep. 2016;6:32252.

40. Sibthorp C, Wu H, Cowley G, Wong PWH, Palaima P, Morozov IY, et al. Transcriptome analysis of the filamentous fungus Aspergillus nidulans directed to the global identification of promoters. BMC Genomics. 2013;14:847.

41. Abraham K, Ostrowski LA, Mekhail K. Non-coding RNA molecules connect calorie restriction and lifespan. J Mol Biol. 2016; doi: 10.1016/j. jmb.2016.08.020.

42. Chacko N, Zhao Y, Yang E, Wang L, Cai JJ, Lin X. The IncRNA RZE1 controls Cryptococcal morphological transition. Plos Genet. 2015;11:e1005692.

43. Xue Z, Ye Q, Anson SR, Yang J, Xiao G, Kowbel D, et al. Transcriptional interference by antisense RNA is required for circadian clock function. Nature. 2014:514:650-3.

44. Li N, Joska TM, Ruesch CE, Coster SJ, Belden WJ. The frequency natural antisense transcript first promotes, then represses, frequency gene expression via facultative heterochromatin. Proc Natl Acad Sci U S A. 2015;112:4357-62.

45. Dang Y, Cheng J, Sun X, Zhou Z, Liu Y. Antisense transcription licenses nascent transcripts to mediate transcriptional gene silencing. Genes Dev. 2016; doi: 10.1101/gad.285791.116.

46. Cho Y-B, Lee EJ, Cho S, Kim TY, Park JH, Cho B-K. Functional elucidation of the non-coding RNAs of Kluyveromyces marxianus in the exponential growth phase. BMC Genomics. 2016;17:154

47. Ponting CP, Oliver PL, Reik W. Evolution and functions of long noncoding RNAs. Cell. 2009;136(4):629-41.

48. Donaldson ME, Meng S, Gagarinova A, Babu M, Lambie SC, Swiadek AA, et al. Investigating the ustilago maydis/Zea mays pathosystem: transcriptional responses and novel functional aspects of a fungal calcineurin regulatory $B$ subunit. Fungal Genet Biol. 2013;58-59:91-104.

49. Zahiri AR, Babu MR, Saville BJ. Differential gene expression during teliospore germination in Ustilago maydis. Mol Genet Genomics. 2005;273:394-403.

50. Sambrook J, Russell DW. Molecular cloning: a laboratory manual. Cold Spring Harbor: Cold Spring Harbor Laboratory Press; 2001.

51. Lagesen K, Hallin P, Rødland EA, Stærfeldt H-H, Rognes T, Ussery DW. RNAmmer: consistent and rapid annotation of ribosomal RNA genes. Nucleic Acids Res. 2007:35:3100-8.

52. Petersen TN, Brunak S, von Heijne G, Nielsen H. SignalP 4.0: discriminating signal peptides from transmembrane regions. Nat Methods. 2011;8:785-6.

53. Donaldson ME, Saville BJ. Bioinformatic identification of Ustilago maydis meiosis genes. Fungal Genet Biol. 2008;45 Suppl 1:S47-53.

54. Conesa A, Götz S, García-Gómez JM, Terol J, Talón M, Robles M. Blast2GO: a universal tool for annotation, visualization and analysis in functional genomics research. Bioinformatics. 2005;21:3674-6.

55. Reimand J, Arak T, Adler P, Kolberg L, Reisberg S, Peterson H, et al. g: Profiler-a web server for functional interpretation of gene lists. Nucleic Acids Res. 2016; doi: 10.1093/nar/gkw199. 
56. Mcginnis S, Madden TL. BLAST: at the core of a powerful and diverse set of sequence analysis tools. Nucleic Acids Res. 2004;32:W20-5.

57. Mewes HW, Dietmann S, Frishman D, Gregory R, Mannhaupt G, Mayer KF, et al. MIPS: analysis and annotation of genome information in 2007. Nucleic Acids Res. 2008;36:D196-201.

58. Ho EC, Donaldson ME, Saville BJ. Detection of antisense RNA transcripts by strand-specific RT-PCR. Methods Mol Biol. 2010;630:125-38.

59. Holliday R. The genetics of Ustilago maydis. Genet Res. 1961;2:204-30.

60. Linning R, Lin D, Lee N, Abdennadher M, Gaudet D, Thomas P, et al. Markerbased cloning of the region containing the UhAvr1 avirulence gene from the basidiomycete barley pathogen Ustilago hordei. Genetics. 2004;166:99-111.

61. Schirawski J, Heinze B, Wagenknecht M, Kahmann R. Mating type loci of Sporisorium reilianum: novel pattern with three a and multiple b specificities. Eukaryot Cell. 2005;4:1317-27.

Submit your next manuscript to BioMed Central and we will help you at every step:

- We accept pre-submission inquiries

- Our selector tool helps you to find the most relevant journal

- We provide round the clock customer support

- Convenient online submission

- Thorough peer review

- Inclusion in PubMed and all major indexing services

- Maximum visibility for your research

Submit your manuscript at www.biomedcentral.com/submit
) Biomed Central 